\title{
Spinal cord compression due to extramedullary haemopoiesis in myelofibrosis
}

\author{
G Cook, R A Sharp
}

\begin{abstract}
Extramedullary haemopoiesis resulting in spinal cord compression is rare. This report of extramedullary myeloid metaplasia in a patient with myelofibrosis serves to illustrate the value of magnetic resonance imaging (MRI) in the diagnosis and management of good neurological recovery.
\end{abstract}

(f Clin Pathol 1994;47:464-465)

Extramedullary haemopoiesis (EMH) or myeloid metaplasia is associated with many chronic haematological conditions, including
Postoperative recovery was uncomplicated. The patient received megavoltage radiotherapy to the site of maximal compression with an incident dose of $2500 \mathrm{cGy}$, delivered in five daily doses over one week which was well tolerated. This was followed by intensive physiotherapy. Follow up at six weeks and six months showed steady neurological improvement and improved mobility.

\section{Discussion}

Extramedullary haemopoiesis is a well described complication of many chronic haematological conditions. This haemopoietic tissue is rarely seen in sites other than those that normally occur in the foetus (table). Rarely, EMH has been found localised in the dura mater of the spinal cord, and can result in spinal cord compression. This was first reported in 1956 in a patient with myelofibrosis. ${ }^{2}$ Since then there have been more than 50 such reported cases, most of which occurred in association with thalassaemia. ${ }^{3}$ The dura mater has probably been involved more than this implies as this region is rarely, if ever, examined at necropsy. The exact origin of EMH in the dura mater is unknown, but as indicated the dura mater has haemopoietic capacity in the foetus and $\mathrm{EMH}$ may develop from primitive rests. An alternative explanation is the embolisation of haemopoietic stem cells to the dura mater. ${ }^{4}$ It is unlikely, however, that EMH arises from extrusion of vertebral bone marrow in the absence of bony erosions or fractures.

In spinal cord compression secondary to $\mathrm{EMH}$, the lesions are commonly localised to the mid-lower thoracic region. This may be because of the narrow diameter of the

Sites in which extramedullary haemopoiesis has been documented, in order of frequency ${ }^{2}$

\begin{tabular}{|c|c|}
\hline & 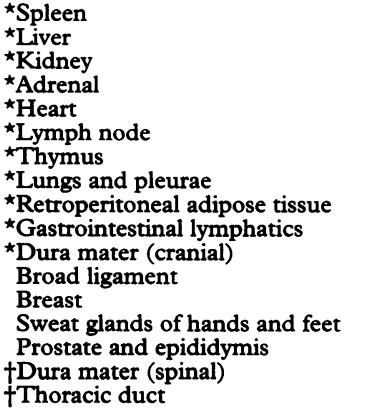 \\
\hline
\end{tabular}

* Sites described in embryo and neonate. $\nmid$ Sites described in embryo only. \begin{abstract}
thalassaemia, leukaemia, lymphoma, and myelofibrosis. ${ }^{1}$ It most commonly affects the liver, spleen, kidneys, and adrenal glands. Involvement of the dura mater of the spinal cord, resulting in spinal cord compression, has occasionally been described. We report a case of spinal cord compression due to EMH in association with myelofibrosis.

\section{Case report}

A 67 year old man had hepatosplenomegaly and a leucoerythroblastic peripheral blood film. Bone marrow examination confirmed the diagnosis of primary myelofibrosis. A splenectomy was carried out four months later for persisting left upper quadrant abdominal discomfort.

He remained well postoperatively with minimal transfusion requirements, although platelet counts did not rise above $20 \times 10^{9} / 1$. One year later, he presented with heaviness of both lower limbs but formal neurological assessment showed no abnormality. Over the next nine months his mobility deteriorated. Formal neurological examination at this point showed diminished power, exaggerated deep tendon reflexes, and bilateral ankle clonus. A sensory level was detected in the mid-thoracic (T12) region. There was no functional urinary disturbance.

Chest and thoracolumbar radiographs were normal. Balanced sagittal magnetic resonance imaging (MRI) showed an extensive extradural mass from the upper cervical to lower lumbar area with maximal depth at the T6-T7 level (fig 1). Partial laminectomy and biopsy was performed with transfused platelet cover. Histological examination showed abundant fibrotic haemopoietic tissue, including numerous myeloid precursors and megakaryocytes (fig 2).

illustrates extramedullary mass $(A)$ at maximal displacing the spinal cord (C) anteriorly.

Figure 1 Balanced

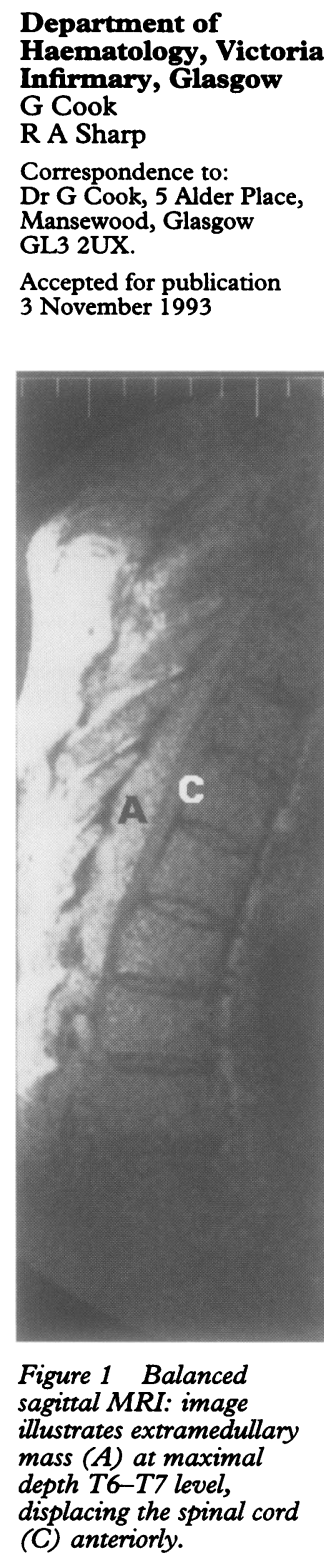




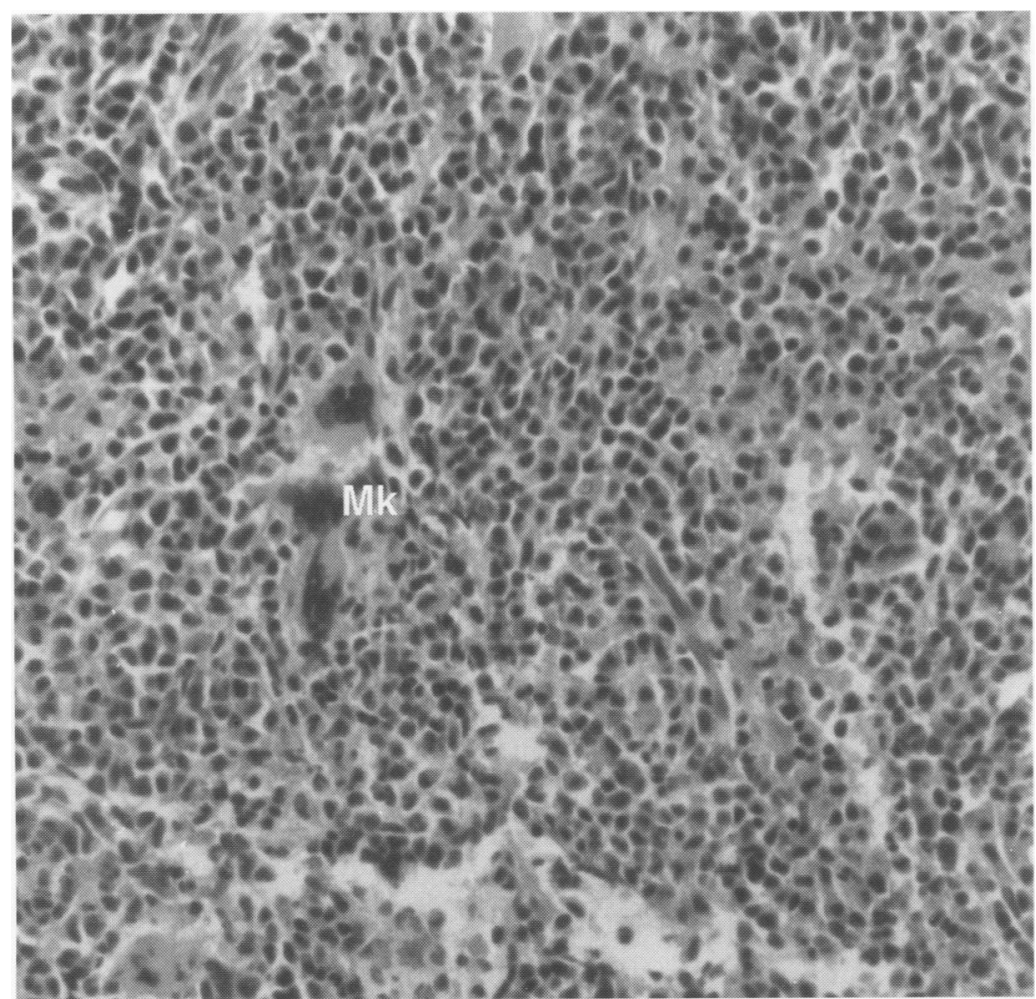

Figure 2 Biopsy specimen of extradural mass revealing cellular tissue of haemopoietic origin with clusters of megakaryocytes (Mk) (haematoxylin and eosin).

spinal cord in this region. ${ }^{4}$ In earlier reports, diagnosis was made by myelography followed by decompression laminectomy and histological analysis of material gained. This served as both a diagnostic and therapeutic procedure. Although surgery is effective in most cases, haemopoietic tissue is radiosensitive and radiotherapy was introduced as an adjunct to surgery either because of incomplete excision or persisting neurological deficit. This dual approach was documented in several of the earlier cases, with satisfactory responses in most patients. ${ }^{56}$

More recently this combined approach has been challenged with the advent of more complex radiological imaging techniques and by the use of radiotherapy alone. One group, the first to report the use of MRI in the diagnosis of spinal cord compression secondary to EMH, reported five patients with thalassaemia who responded to radiotherapy alone..$^{5}$ This approach has the advantage of avoiding a major surgical procedure in patients whose risk of haemorrhage is compounded by thrombocytopenia, as in our patient, particularly when the mass of EMH is diffuse and complete resection is impossible. ${ }^{6}$

The dose of radiation used has varied from 900 cGy to 3500 cGy with different fraction sizes. ${ }^{57}$ With the availability of MRI the radiation ports can be delineated more accurately to limit the extent of spinal cord at risk and thus low dose radiotherapy can be used to achieve a satisfactory clinical response. ${ }^{8}$ Recovery was prompt in most cases reported, beginning within a few days.

This case shows how MRI imaging can facilitate the diagnosis and assessment of the extent of extramedullary myeloid metaplasia in a patient with primary myelofibrosis leading to spinal cord compression.

We thank Dr $M$ Millar (consultant radiologist), Mr R A Johnston (consultant neurosurgeon), and Dr J Nicoll (senior lecturer in neuropathology) for their assistance with this case.

1 Ward HP, Block MH. The natural history of agnogenic myeloid metaplasia \& a critical evaluation of its relationship with the myeloproliferative syndrome. Medicine 1971;50:357-411.

2 Close AS, Taira Y, Cleveland DA. Spinal cord compression due to extramedullary haematopoiesis. Ann Intern Med 1958;48:421-7.

3 Singhal S, Sharma S, Dixit S, De S, Chander S, Rath GK, Mehta VS. The role of radiation therapy in the management of spinal cord compression due to extramedullary haematopoiesis in thalassaemia. 7 Neurol Neurosurg Psychiatry 1993;55:310-12.

4 Heffner RR, Koehl RH. Haematopoiesis in the spinal epidural space. $\mathcal{F}$ Neurosurg 1970;32:485-8.

5 Papvasilou C, Sandilos P. Effect of radiotherapy on symptoms due to heterotropic marrow in $\beta$-thalassaemia. Lancet 1987;1:13-14.

6 Oustwani MB, Kurtides ES, Christ M, Ciric I. Spinal cord compression with paraplegia in myelofibrosis. Arch Neurol 1980;37:389-90.

7 Issaragrisil S, Piankijagoni A, Wasi P. Spinal cord compression due to extramedullary haemopoiesis in thalassaemia: longterm follow-up after radiotherapy. Surg Neurol 1988;29:389-92.

8 Hassoun H, Lawn-Tsao L, Langerin ER, Lathi ES, Palek J. Spinal cord compression secondary to extramedullary haemopoiesis: a non-invasive management based on haemopoiesis: a non-invasive man 